\title{
HOMEOMORPHISMS BETWEEN BANACH SPACES
}

\author{
BY
}

\section{ROY PLASTOCK( $(1)$}

\begin{abstract}
We consider the problem of finding precise conditions for a map $F$ between two Banach spaces $X, Y$ to be a global homeomorphism.

Using methods from covering space theory we reduce the global homeomorphism problem to one of finding conditions for a local homeomorphism to satisfy the "line lifting property." This property is then shown to be equivalent to a limiting condition which we designate by (L). Thus we finally show that a local homeomorphism is a global homeomorphism if and only if (L) is satisfied. In particular we show that if a local homeomorphism is

(i) proper (Banach-Mazur) or

(ii) $\int_{0}^{\infty} \inf _{\|x\|<s} 1 /\left\|\left[F^{\prime}(x)\right]^{-1}\right\| d s=\infty$ (Hadamard-Levy), then (L) is satisfied. Other analytic conditions are also given.
\end{abstract}

Introduction. Suppose we have a continuous (or continuously differentiable) map $F$ between two Banach spaces $X$ and $Y$. We ask what additional assumptions must be imposed upon $F$ to insure that it is a homeomorphism (diffeomorphism) of $X$ onto $Y$ ? We observe that if $F$ is a homeomorphism then in particular it is a local homeomorphism. Also if $F$ is a diffeomorphism, then by the chain rule $F^{\prime}(x)$ is an invertible linear map for every $x$. Since these conditions are necessary for our problem, we shall always assume that our map $F$ is either a local homeomorphism or, if $F \in C^{1}(X)$, that $F^{\prime}(x)$ is invertible. Thus we ask when such a map is a homeomorphism (diffeomorphism) of $X$ onto $Y$.

Using Lemma 1.1 we shall see that we can reduce our problem to a more fundamental one: that of determining when a given local homeomorphism $F$ is a covering space map of $X$ onto $Y$. In $\S 1$ we present the following solution to this question (Theorem 1.1):

If $F: D \subseteq X \rightarrow Y$ is a local homeomorphism (where $X$ and $Y$ are Banach spaces, $D$ open), then $(D, F)$ is a covering space of $F(D)$ if and only if $F$ "lifts lines."

We next introduce a simple analytic criterion, condition $(\mathrm{L})$, which insures

Received by the editors January 18, 1973 and, in revised form, May 8, 1973.

AMS (MOS) subject classifications (1970). Primary 47H99, 54C99, 55A10.

Key words and phrases. Covering space.

(1) Research partially supported by the AFOSR. 
that $F$ lifts lines. More precisely, we show (Theorem 1.2) that a map is a global homeomorphism if and only if it is a local homeomorphism and satisfies condition (L). Thus, in view of Theorem 1.2, we are led to determine precise conditions which insure that a map satisfies condition (L).

In $\S 2$ we impose conditions which lead to direct consequences of Theorem 1.2, the simplest being the conditions of Banach and Mazur: $F: X \rightarrow Y$ is a homeomorphism if and only if $F$ is a local homeomorphism and a proper map.

Our investigation continues in $\S 3$ in the spirit of the Hopf-Rinow theorem of differential geometry. Here we give a definition of completeness in a Banach space setting which enables us to verify condition $(\mathrm{L})$ under various analytic conditions imposed on a map $F$. Global homeomorphism theorems are proven for a special class of quasi-conformal maps (see Zoric [16]) and also the Hadamard-Levy theorem is deduced as a special case of Theorem 3.1 (which gives analytic criteria that insures completeness according to Definition 3.2).

$\S 4$ is devoted to global homeomorphisms between finite-dimensional Euclidean spaces. In particular, if $\mid \mathrm{J}$ acobian $F \mid>\alpha>0$, what additional assumptions must be imposed upon $F$ to insure that it is a global homeomorphism? Several answers are given using the Hadamard-Levy theorem.

The background references for this chapter are [1], [2], [4], [5], [6], [8], $[9],[10],[12],[13],[14],[16]$. Further references can be found in these papers.

1. Local homeomorphisms and covering spaces. Our approach for attacking the global homeomorphism problem is motivated by the following well-known result:

LEMMA 1.1. Let $X$ and $Y$ be connected, locally pathwise connected spaces. Furthermore, let $Y$ be simply connected. Then $F$ is a homeomorphism of $X$ onto $Y$ if and only if $(X, F)$ is a covering space of $Y$.

In order to apply Lemma 1.1 to our problem we must find precise conditions for determining when a local homeomorphism is a covering space map.

Let $X$ and $Y$ be Banach spaces, $D \subseteq X$ open and connected.

DEFinition 1.1. $F: D \rightarrow Y$ lifts lines in $F(D)$ if and only if for each line $L(t)=(1-t) y_{1}+t y_{2}$ in $F(D)$ and for every point $x_{\alpha} \in F^{-1}\left(y_{1}\right)$ there is a path $P_{\alpha}(t)$ such that $P_{\alpha}(0)=x_{\alpha}$ and $F\left(P_{\alpha}(t)\right)=L(t)$.

A simple connectedness argument shows that if $F$ is a local homeomorphism and $F$ lifts lines in $F(D)$, then the path $P_{\alpha}(t)$ in Definition 1.1 is unique.

Theorem 1.1. Let $D \subseteq X$ be open and connected, $F: D \rightarrow X$. The following are necessary and sufficient conditions for $(D, F)$ to cover $F(D)$ : 
(i) $F$ is a local homeomorphism, and

(ii) $F$ lifts lines in $F(D)$.

Proof. The necessity follows from the properties of a covering space. To prove the sufficiency, we first observe that if $y \in F(D)$, we can find an $r$ so that $B(y ; r)=\{z \mid\|z-y\|<r\} \subseteq F(D)$, and that any radius in $B$ can be described by a line $L_{z}(t)=y+\operatorname{tr} z,\|z\|=1,0 \leqslant t<1$, which can be lifted. Let $x \in F^{-1}(y)$,

$$
O_{x}^{*}=\left\{P(t) \mid F(P(t))=L_{z}(t), \mathrm{V}\|z\|=1,0 \leqslant t<1 \text {, and } P(0)=x\right\} .
$$

Let $O_{x}=O_{x}^{*}$ considered as a point set, i.e., $O_{x}=\left\{x \mid x=P(\bar{t}), P \in O_{x}^{*}\right\}$ $\left(O_{x} \neq \varnothing\right.$ by (ii)). By intuitively thinking of $O_{x}$ and $B(y ; r)$ as the spokes of a wheel, we shall show that these sets satisfy the conditions given in the definition of a covering space, i.e., we show that the $O_{x}\left(x \in F^{-1}(y)\right)$ are disjoint, open sets mapped homeomorphically onto $B(y ; r)$ by $F$, and $F^{-1}(B(y ; r))=$ $\bigcup_{x \in F^{-1}(y)} O_{x}$.

(a) Each $O_{x}$ is mapped onto $B(y ; r)$ since any $\bar{y} \in B(y ; r)$ lies on some radius $L$; hence there is a path $P(t) \in O_{x}^{*}$ and a $\bar{t}$ so that $F(P(\bar{t}))$ $=\bar{y}$. By definition of $O_{x}, P(\bar{t}) \in O_{x}$.

(b) Each $O_{x}$ is mapped homeomorphically onto $B(y ; r)$. If not, let $x_{1} \neq$ $x_{2} \in O_{x}$ and $F\left(x_{1}\right)=F\left(x_{2}\right)=\bar{y}$. By definition of $O_{x}, x_{1}$ and $x_{2}$ lie on paths $P_{1}$ and $P_{2}$ which are not identical, for otherwise their image would be a radius which would intersect itself. Hence $F\left(P_{1}(t)\right)$ and $F\left(P_{2}(t)\right)$ are distinct radii. Thus $\bar{y}=y$, and so $F\left(x_{1}\right)=F\left(P_{1}\left(t_{1}\right)\right)=F(P(0))=y=F\left(P_{2}\left(t_{2}\right)\right)=$ $F\left(x_{2}\right)$. Hence $t_{1}=0$ and $t_{2}=0$ (otherwise the image of $P_{i}(t)(i=1,2)$ would be a radius which intersects itself), and so $x_{1}=x_{2}=x$, a contradiction. The continuity of the inverse will follow from the fact that $\left.F\right|_{o_{x}}$ is a local homeomorphism and thus an open map (see (d) below).

(c) $O_{x}, x \in F^{-1}(y)$, are disjoint, for if $\bar{x} \in O_{x_{1}} \cap O_{x_{2}}$ with $x_{1} \neq x_{2}$, then $\bar{x}=P_{1}\left(t_{1}\right)=P_{2}\left(t_{2}\right)$. The images of $P_{1}$ and $P_{2}$ under $F$ must be the same radius, for otherwise the radii would intersect and so $F(\bar{x})=F\left(x_{1}\right)=$ $F\left(x_{2}\right)=y$. By part (b), $\bar{x}=x_{1}=x_{2}$-a contradiction. Thus $F\left(P_{1}(t)\right)=$ $F\left(P_{2}(t)\right)=L(t)$, and so $L\left(t_{1}\right)=L\left(t_{2}\right)$ which implies that $t_{1}=t_{2}(=\bar{t})$. Hence we conclude that $P_{1}(t)=P_{2}(t)$ and, in particular, $x_{1}=x_{2}$. Thus the $O_{x}$ 's are disjoint.

(d) Each $O_{x}$ is an open set in $D$. For if $u \in O_{x}, u \neq x$, then there is a path $P(t)(0 \leqslant t \leqslant 1)$ in $O_{x}$ with initial point $x$ and terminal point $u$ such that $F(P(t))=(1-t) y+t v$ (where $y=F(x), v=F(u)$ ).

By (b) above, $F$ is one-one on $P(t)$. This together with the compactness of the path $P(t)$ enables us to find an open set $S$ containing $P(t)$ so that $F$ 
is a homeomorphism on $S$ to the open set $F(S)$. To show that $u$ is an interior point of $O_{x}$, we first find an open ball $B(v ; \delta) \subseteq F(S)$ with the property that the line $(1-t) y+t w$ is in $F(S)$ whenever $w$ is in $B(v ; \delta)$. If this were not the case we would find sequences $t_{i}\left(0 \leqslant t_{i} \leqslant 1\right)$ and $w_{i} \rightarrow v$ so that $\left(1-t_{i}\right) y$ $+t_{i} w_{i}=y_{i} \notin F(S)$. However a suitable subsequence of the $y_{i}$ converges to a point $(1-\bar{t}) y+\bar{t}$ which is in $F(S)$, thus contradicting the openness of $F(S)$. Thus $F^{-1}(B(v ; \delta)) \cap S$ is an open set in $O_{x}$ containing $u$. The interiorness of the remaining case $u=x$ follows directly from hypothesis (i).

The openness of $O_{x}$ and hypothesis (i) implies that $\left.F\right|_{O_{x}}$ is a local homeomorphism.

(e) $F^{-1}(B(y ; r))=\bigcup_{x \in F^{-1}(y)} O_{x}$.

Since $F^{-1}(B(y ; r)) \subseteq \bigcup_{x \in F^{-1}(y)} O_{x}$, it suffices to show the opposite inclusion. So let $x \in F^{-1}(B(y ; r))$. Let $L(t)=(1-t) F(x)+t y, 0 \leqslant t \leqslant 1$. Then $L(t) \in B(y ; r)$ and so by hypothesis there is a path $P(t)$ so that $P(0)=x$ and $F(P(t))=L(t)$. Thus $P(1) \in F^{-1}(y)$. Let $L^{\prime}(t)=L(1-t)$ and $P^{\prime}(t)=$ $P(1-t)$. Thus $F\left(P^{\prime}(t)\right)=L^{\prime}(t), P^{\prime}(0)=P(1) \in F^{-1}(y)$ and $P^{\prime}(1)=x$. So, by definition of $O_{P(1)}$, we see that $x \in O_{P(1)}$.

We wish to remark that, for finite-dimensional spaces, Theorem 1.1 is essentially due to Hermann [17, pp. 286-290].

In view of Theorem 1.1 we now proceed in developing a method (Theorem 1.2) for determining when a local homeomorphism lifts lines.

Again we suppose that $X$ and $Y$ are Banach spaces, $D \subseteq X$ is open and connected. Let $F: D \rightarrow Y$ be continuous. We introduce the following condition:

(L) Whenever $P(t), 0 \leqslant t<b$, is a path satisfying $F(P(t))=L(t)$ for $0 \leqslant t<b$ (where $L(t)=(1-t) y_{1}+t y_{2}$ is any line in $Y$ ), then there is a sequence $t_{i} \rightarrow b$ as $i \rightarrow \infty$ such that $\lim _{i \rightarrow \infty} P\left(t_{i}\right)$ exists and is in $D$.

THEOREM 1.2. Let $F: D \subseteq X \rightarrow Y$ be a local homeomorphism. Then condition $(\mathrm{L})$ is necessary and sufficient for $F$ to be a homeomorphism of $D$ onto $Y$.

Proof. The necessity is trivial, for we let $P(b)=F^{-1}(L(b))$. For the sufficiency, we first show that $F$ lifts lines. Let $L(t)$ be any line in $F(D)$, with $L(0)=\bar{y}$. Let $\bar{x} \in F^{-1}(\bar{y})$.

Since $F$ is a local homeomorphism, there is an $\epsilon>0$ and a path $P(t)$ $\left(=F^{-1}(L(t))\right), 0 \leqslant t<\epsilon$, such that $P(0)=\bar{x}$ and $F(P(t))=L(t)$ for $0 \leqslant$ $t<\epsilon$. Let $K(\leqslant 1)$ be the largest number for which $P(t)$ can be extended to a continuous path for $0 \leqslant t<K$ and satisfying $F(P(t))=L(t), 0 \leqslant t<K$. Since $F$ satisfies condition (L), let $z=\lim _{t_{i} \rightarrow K} P\left(t_{i}\right)$. By continuity, $F(z)=$ $L(K)$. Let $W$ be a neighborhood of $z$ on which $F$ is a homeomorphism. $3 N$ so that $P\left(t_{i}\right) \in W$ for $i \geqslant N$. Also a $\delta>0$ and a path $Q(t)$ defined for 
$K-\delta<t<K+\delta$ so that $Q\left(t_{M}\right)=P\left(t_{M}\right)$ (where $M$ is chosen so that $M \geqslant N$ and $\left.K-\delta<t_{M}<K\right)$ and $F(Q(t))=L(t)$ for $K-\delta<t<K+\delta$.

Hence $P(t)$ can be extended to a continuous path (which we again call $P(t))$ on $0 \leqslant t<K+\delta, P(0)=\bar{x}$ and $F(P(t))=L(t), 0 \leqslant t<K+\delta$. By the maximality of $K$, we conclude that $K=1$, and hence $F$ lifts lines.

By virtue of Theorem 1.1, $(D, F)$ covers $F(D)$. We need only show that $F(D)=Y$ in order to apply Lemma 1.1 and thus conclude that $F$ is a homeomorphism of $D$ onto $Y$. So let $\bar{y} \in Y$. Choose $y_{1} \in F(D)$ and let $L(t)=$ $(1-t) y_{1}+t \bar{y}$. If we retrace the steps of the first part of our proof, we find a path $P(t), 0 \leqslant t \leqslant 1$, so that $F(P(t))=L(t)$ on $0 \leqslant t \leqslant 1$. In particular, $F(P(1))=L(1)=\bar{y}$, and so $F(D)=Y$.

2. Direct consequences of Theorem 1.2. In this section we impose precise conditions upon a map $F$ between two Banach spaces which lead to straightforward verifications of condition (L). An application of Theorem 1.2 will then yield the desired global homeomorphism result.

Theorem 2.1. Let $F: X \rightarrow Y$ be continuously differentiable and also $F^{\prime}(x)$ is invertible for all $x \in X$. Suppose that

(i) $\|F(x)\| \rightarrow \infty$ as $\|x\| \rightarrow \infty$,

(ii) $\left\|\left[F^{\prime}(x)\right]^{-1}\right\| \leqslant M(\|x\|)$, where $M(t)$ is a continuous positive function of $\mathbf{R} \rightarrow \mathbf{R}$.

Then $F$ is a diffeomorphism of $X$ onto $Y$.

Proof. By Theorem 1.2, it suffices to show that $F$ satisfies condition (L). By the construction of Theorem 1.2 and the inverse function theorem, it suffices to show that condition (L) is satisfied for paths $P(t)$ which are continuously differentiable on $0 \leqslant t<b$. So suppose $P(t)$ is defined on $0 \leqslant t<b$, is continuously differentiable and satisfies

$$
F(P(t))=L(t)\left(=(1-t) y_{1}+t y_{2}\right) \text { for } 0 \leqslant t<b .
$$

By (i), $S=F^{-1}(L(t))_{0<t<1}$ is a bounded set, and so $\{P(t)\}_{0<t<b}$ is bounded. Since $P(t)$ is continuously differentiable, we can apply the chain rule to (1) and thus

$$
F^{\prime}(P(t)) P^{\prime}(t)=y_{2}-y_{1}=z, \quad 0 \leqslant t<b .
$$

Therefore $P^{\prime}(t)=\left[F^{\prime}(P(t))\right]^{-1} z, 0 \leqslant t<b$. By (ii), aC so that $\left\|\left[F^{\prime}(P(t))\right]^{-1}\right\|$ $\leqslant C$ for $0 \leqslant t<b$. Let $t_{i} \rightarrow b$.

$$
P\left(t_{M}\right)-P\left(t_{N}\right)=\int_{t_{N}}^{t_{M}} P^{\prime}(t) d t=\int_{t_{N}}^{t_{M}}\left[F^{\prime}(P(t))\right]^{-1} z d t .
$$




$$
\left\|P\left(t_{M}\right)-P\left(t_{N}\right)\right\| \leqslant \int_{t_{N}}^{t_{M}}\left\|\left[F^{\prime}(P(t))\right]^{-1}\right\|\|z\| d t \leqslant C\|z\|\left|t_{M}-t_{N}\right| .
$$

Thus $\left\{P\left(t_{i}\right)\right\}$ is a Cauchy sequence, and so condition (L) is verified, as was to be shown.

The next theorem is originally due to Banach and Mazur [1].

Definition 2.1. A continuous map $F$ between two topological spaces $X$ and $Y$ is proper if and only if $F^{-1}(C)$ is a compact set in $X$ whenever $C$ is a compact set in $Y$.

Theorem 2.2. Let $X$ and $Y$ be Banach spaces, $F: X \rightarrow Y$. Then $F$ is a homeomorphism of $X$ onto $Y$ if and only if $F$ is a local homeomorphism and a proper map.

Proof. The necessity is obvious for if $F$ is a homeomorphism, then $F^{-1}$ is continuous and thus maps compact sets into compact sets. Hence $F$ is proper.

Suppose now that $F$ is a local homeomorphism and $F$ is proper. By virtue of Theorem 1.2, it suffices to show that $F$ satisfies condition (L) in order to conclude that $F$ is a homeomorphism. So suppose $P(t)$ is defined on $0 \leqslant t$ $<b$ and satisfies $F(P(t))=L(t)$ for $0 \leqslant t<b$. Let $t_{i} \rightarrow b$. Since $S=$ $\{L(t)\}_{0<t<1}$ is compact, so is $F^{-1}(S)$ and it contains the sequence $P\left(t_{i}\right)$. Hence there is a subsequence $t_{i_{j}} \rightarrow b$ such that $P\left(t_{i_{j}}\right) \rightarrow \bar{x}$, and so condition $(\mathrm{L})$ is satisfied.

Corollary 2.1. $F: \mathbf{R}^{N} \rightarrow \mathbf{R}^{N}$ is a diffeomorphism if and only if $F \in$ $C^{1}\left(\mathrm{R}^{N}\right)$ and $F$ satisfies (i) $\operatorname{det} F^{\prime}(x) \neq 0 \forall \dot{x}$, and (ii) $\|F(x)\| \rightarrow \infty$ as $\|x\| \rightarrow \infty$.

Proof. We observe that if $F$ is a diffeomorphism, then an application of the chain rule shows that $F^{\prime}(x)$ is invertible and thus $\operatorname{det} F^{\prime}(x) \neq 0$. Also, (ii) is equivalent to the condition that $F^{-1}(B)$ is a bounded set whenever $B$ is a bounded set. This in turn is equivalent, for continuous maps between finitedimensional Banach spaces, to properness. Thus the proof of the corollary follows immediately from Theorem 2.2 .

It is interesting to note that if (i) is replaced by the weaker hypothesis $\left(\mathrm{i}^{*}\right)$ $\operatorname{det} F^{\prime}(x) \geqslant 0(\neq 0)$, then (i*) and (ii) imply that $F$ maps $\mathbf{R}^{N}$ onto itself (in general, such a map will not be one-one). The proof of this remark, along with further generalizations, is to be found in [15].

COROLLARY 2.2. Let $F$ be a local homeomorphism of the reflexive space $X$ into $Y$. If (i) $\|F(x)\| \rightarrow \infty$ as $\|x\| \rightarrow \infty$ and (ii) whenever $x_{n} \rightarrow x$ weakly, and $F\left(x_{n}\right) \rightarrow y$ strongly implies $x_{n} \rightarrow x$ strongly, then $F$ is a homeomorphism of $X$ onto $Y$. 
Proof. We shall show that (i) and (ii) imply that $F$ is proper. The proof of the corollary is then a consequence of Theorem 2.2.

Let the sequence $x_{n} \in F^{-1}(C), C$ a compact set in $Y$. By (i), $F^{-1}(C)$ is bounded. Since $X$ is reflexive there is a subsequence (after renumbering) $x_{n}$ which converges weakly to some $x$. As $C$ is compact, we may assume (by passing to an appropriate subsequence and renumbering) that the sequence $F\left(x_{n}\right)$ converges strongly to some $y \in C$. From (ii) we conclude that $x_{n}$ converges strongly to $x$. By closedness, $x \in F^{-1}(C)$ and so $F^{-1}(C)$ is compact as was to be shown.

The following theorem is due to Browder [4]:

THEOREM 2.3. $F: X \rightarrow Y$ is a homeomorphism of $X$ onto $Y$ if and only if $F$ is a local homeomorphism and a closed map.

Proof. To prove the necessity we need only notice that if $F$ is a homeomorphism; then $F^{-1}$ is continuous and so $F$ maps closed sets into closed sets.

To prove the sufficiency, we need only show that $F$ satisfies condition (L) and then apply Theorem 1.2 to conclude that $F$ is a homeomorphism of $X$ onto $Y$.

We now show that $F$ satisfies condition (L). So suppose that $P(t)$ is defined on $0 \leqslant t<b$ and satisfies $F(P(t))=L(t)$ for $0 \leqslant t<b$. Let $S=$ ${\overline{\{P(t)\}_{0<t<b}}}$. By hypothesis $F(S)$ is closed. Thus since $L(t) \in F(S)$, for all $t<b$, then by continuity $L(b) \in F(S)$. Hence $\mathrm{A} x \in S$ so that $F(x)=L(b)$. Since $x \in S, \exists t_{i}$ so that $P\left(t_{i}\right) \rightarrow x$. Since $0 \leqslant t_{i}<b$, there exists a subsequence $t_{i_{j}} \rightarrow \bar{t}$. We claim $\bar{t}=b$ (and thus condition (L) is satisfied by $t_{i_{j}} \rightarrow$ $b$ and $\left.P\left(t_{i_{j}}\right) \rightarrow x\right)$. However, by continuity, $L(\bar{t})=L(b)$ and so $\bar{t}=b$.

Let us remark that since a proper map is in particular a closed map, we could have deduced Theorem 2.2 as a corollary of Theorem 2.3. However, we preferred to prove it directly in order to illustrate the type of arguments that one can use in verifying condition (L).

Corollary 2.3. Suppose $F: X \rightarrow Y$ is a local homeomorphism. Furthermore, suppose $F$ satisfies the following:

(1) $\|F(x)\| \rightarrow \infty$ as $\|x\| \rightarrow \infty$.

(2) There exists a compact operator $K: X \rightarrow Y$ such that the operator $B(x)=F(x)+K(x)$ satisfies the following condition: For any $x_{1}$ and $x_{2}$ with $\left\|x_{1}\right\|$ and $\left\|x_{2}\right\| \leqslant R$ we have $\left\|B\left(x_{2}\right)-B\left(x_{1}\right)\right\| \geqslant \phi\left(\left\|x_{2}-x_{1}\right\| ; R\right)$, where $\phi(r ; R)$ is continuous, real-valued and strictly increasing with respect to $r \geqslant 0$ for each $R>0$ and $\phi(0 ; R) \equiv 0$.

If the above conditions are satisfied, then $F$ is a homeomorphism of $X$ onto $Y$. 
Proof. We shall show that $F$ is a closed map and then use Theorem 2.3 to conclude the desired result. Let $C \subseteq X$ be closed, and let the sequence $x_{i} \in C$ be such that $F\left(x_{i}\right) \rightarrow y$. By (1), the sequence $x_{i}$ is bounded (by $M$ ) and since $K$ is compact, there is a subsequence $x_{n_{j}}$ such that $K\left(x_{n_{j}}\right) \rightarrow \bar{y}$. Hence $B\left(x_{n_{j}}\right) \rightarrow y+\bar{y}$. Suppose $\exists \epsilon_{0}>0$ such that $\left\|x_{n_{j}}-x_{m_{j}}\right\| \geqslant \epsilon_{0}>0$ for all $n_{j}, m_{j}$. Then $\left\|B\left(x_{n_{j}}\right)-B\left(x_{m_{j}}\right)\right\| \geqslant \phi\left(\left\|x_{n_{j}}-x_{m_{j}}\right\| ; 2 M\right) \geqslant \phi\left(\epsilon_{0}, 2 M\right)>0$, which is a contradiction since $\left\|B\left(x_{n_{j}}\right)-B\left(x_{m_{j}}\right)\right\| \rightarrow 0$. Hence there is a subsequence of $x_{n_{j}}$ which converges to some $x$, and $x \in C$ since $C$ is closed. Thus $F(x)=y$ by continuity, and so $F(C)$ is closed, as was to be shown.

3. Completeness and global homeomorphisms. In differential geometry there is an intimate connection between the continuation of paths of finite length and the completeness of a finite-dimensional manifold with respect to a given metric (Hopf-Rinow theorem [17]).

Since our condition $(\mathrm{L})$ is concerned with the ability of certain paths to have a continuation, we shall formulate a notion of completeness which is suitable for our investigation which takes place in a general Bannach space. As before, Theorem 1.2 will be our main tool in deducing global homeomorphism results.

Again, let $X$ and $Y$ be Banach spaces. Let $B(x)>0$ be a real-valued continuous function on $X$. Let $P(t)$ be a path (in $X$ ) of class $C^{1}$ on $0 \leqslant t \leqslant b$.

Definition 3.1. The arc length of $P$ with weight $B$ is

$$
L_{0}^{b}(P)=\int_{0}^{b} B(P(t))\left\|P^{\prime}(t)\right\| d t
$$

Definition 3.2. $X$ is complete with respect to arc length with weight $B$ if and only if $L_{0}^{b}(P)<\infty \Rightarrow \lim _{t \rightarrow b} P(t)$ exists and is finite whenever $P(t)$ is a $C^{1}$ path on $0 \leqslant t<b$.

We remark that if $X=\mathbf{R}^{N}$, then Definition 3.2 is equivalent to the usual notion of $\mathbf{R}^{N}$ being complete with respect to the conformal metric induced by the tensor $d s^{2}=[B(x)]^{2} d x^{2}$ (see Hartman [11]).

With $B(x)$ as above, we prove the following sufficient condition for completeness:

THEOREM 3.1. Let $h(s)=\inf _{\|x\| \leqslant s} B(x)$. If $\int_{0}^{\infty} h(s) d s=\infty$, then $X$ is complete with respect to arc length with weight $B$.

Proof. Let $P(t) \in C^{1}[0, b)$ and $L_{0}^{b}(P)<\infty$. Let $0<\delta<b$. For any partition $0=t_{0} \leqslant t_{1} \leqslant \cdots \leqslant t_{N}=\delta$ of $[0, \delta]$, let $t_{i} \leqslant \bar{t}_{i} \leqslant t_{i+1}$ be that number for which $\sup _{t_{i} \leqslant t \leqslant t_{i+1}}\left\|P^{\prime}(t)\right\|=\left\|P^{\prime}\left(\bar{t}_{i}\right)\right\|$. By the mean value theorem, we have 


$$
\begin{aligned}
L_{0}^{\delta}(P) & =\int_{0}^{\delta} B(P(t))\left\|P^{\prime}(t)\right\| d t \\
& =\lim \sum B\left(P\left(\bar{t}_{i}\right)\right)\left\|P^{\prime}\left(\bar{t}_{i}\right)\right\|\left(t_{i+1}-t_{i}\right) \\
& \geqslant \lim \sum B\left(P\left(\bar{t}_{i}\right)\right)\left(\left\|P\left(t_{i+1}\right)\right\|-\left\|P\left(t_{i}\right)\right\|\right) \\
& =\int_{0}^{\delta} B(P(t)) d\|P(t)\|,
\end{aligned}
$$

this last equality following from the fact that $\int_{0}^{\delta} B(P(t)) d\|P(t)\|$ is defined since $g(t)=\|P(t)\|$ is of bounded variation on $[0, \delta]$. So we have that

$$
\begin{aligned}
\infty & >\int_{0}^{b} B(P(t))\left\|P^{\prime}(t)\right\| d t \geqslant \int_{0}^{\delta} B(P(t)) d\|P(t)\| \\
& >\int_{0}^{\delta} \inf _{\|x\|<\|P(t)\|} B(x) d\|P(t)\| \\
& =\int_{0}^{\delta} h(\|P(t)\|) d\|P(t)\|=\int_{\|P(0)\|}^{\|P(\delta)\|} h(s) d s .
\end{aligned}
$$

By hypothesis, this implies that $\{P(t)\}_{0<t<b}$ is bounded. Also $\int_{0}^{\infty} h(s) d s=\infty$ implies that $\sup \{s \mid h(s)>0\}=\infty$, and since $h(s)$ is nonincreasing, we have that $B(x)$ is bounded from below on any bounded set. In particular, $B(P(t))$ is bounded from below by some number $\lambda>0$, for all $0 \leqslant t<b$.

Let $t_{i} \rightarrow b,\left(t_{i} \leqslant t_{i+1}\right)$. Then

$$
\begin{aligned}
\sum_{i=1}^{n}\left\|P\left(t_{i+1}\right)-P\left(t_{i}\right)\right\| & \leqslant \sum_{i=1}^{n} \sup _{t_{i}<t<t_{i+1}}\left\|P^{\prime}(t)\right\|\left(t_{i+1}-t_{i}\right) \\
& \leqslant \int_{t_{1}}^{t_{n+1}}\left\|P^{\prime}(t)\right\| d t \leqslant \frac{1}{\lambda} \int_{0}^{b} B(P(t))\left\|P^{\prime}(t)\right\| d t<\infty .
\end{aligned}
$$

Hence $\mathrm{g} \bar{x}$ so that $P\left(t_{i}\right) \rightarrow \bar{x}$ as $t_{i} \rightarrow b$.

Thus $\lim P\left(t_{i}\right)$ exists for any increasing sequence $t_{i} \rightarrow b$, and is in fact unique for such sequences (since from any two such sequences we can form a new increasing sequence containing the original ones as subsequences). Hence $\lim _{t \rightarrow b} P(t)$ exists as every sequence $P\left(t_{i}\right)$ has a unique limit point which is independent of the sequence $t_{i}$.

An immediate consequence of Theorem 3.1 is the following theorem due to Hadamard [10]:

THEOREM 3.2. Let $X$ and $Y$ be Banach spaces, $F: X \rightarrow Y \in C^{1}(X)$ and $F^{\prime}(x)$ is invertible for all $x \in X$. If

$$
\int_{0}^{\infty} \inf _{\|x\|<s} \frac{1}{\left\|\left[F^{\prime}(x)\right]^{-1}\right\|} d s=\infty,
$$

then $F$ is a diffeomorphism of $X$ onto $Y$.

Proof. Since $F$ is a local diffeomorphism, then in view of Theorem 1.2, 
we need only show that $F$ satisfies condition (L). To this end we apply Theorem 3.1 as follows:

Suppose $P(t)$ is defined on $0 \leqslant t<b$ and satisfies $F(P(t))=L(t)$ for $0 \leqslant t<b$. If we look at the proof of Theorem 1.2, we see that it suffices to show that $F$ satisfies condition (L) only for those paths $P(t)$ that are constructed by the method used in Theorem 1.2. Also, if $P(t)$ is such a path, we may assume, by the inverse function theorem, that $P(t)$ is continuously differentiable on $0 \leqslant$ $t<b$. Since $F(P(t))=L(t)$ on $0 \leqslant t<b$, we use the chain rule and get $F^{\prime}(P(t)) P^{\prime}(t)=L^{\prime}(t)(=z)$. Thus $P^{\prime}(t)=\left[F^{\prime}(P(t))\right]^{-1} z$ for $0 \leqslant t<b$. Let $B(x)=1 /\left\|\left[F^{\prime}(x)\right]^{-1}\right\|$. By our hypothesis, combined with Theorem 3.1, we have that $X$ is complete with respect to arc length with weight $B$. Also,

$$
\begin{aligned}
L_{0}^{b}(P) & =\int_{0}^{b} B(P(t))\left\|P^{\prime}(t)\right\| d t \\
& =\int_{0}^{b} \frac{1}{\left\|\left[F^{\prime}(P(t))\right]^{-1}\right\|}\left\|\left[F^{\prime}(P(t))\right]^{-1} z\right\| d t \leqslant b\|z\| .
\end{aligned}
$$

Thus, by Definition 3.2, $F$ satisfies condition (L).

Let us remark that one can use Theorem 2.1 when the integral condition of Theorem 3.2 fails.

CoRollary 3.1. If $F: X \rightarrow Y \in C^{1}(X), F^{\prime}(x)$ is invertible for all $x \in X$ and further there exists $M>0$ so that, for each $x \in X,\left\|F^{\prime}(x) z\right\| \geqslant M\|z\|$ for all $z$, then $F$ is a diffeomorphism of $X$ onto $Y$.

PROOF. $\left\|F^{\prime}(x) z\right\| \geqslant M\|z\|$ for all $z$ implies that $\left\|\left[F^{\prime}(x)\right]^{-1}\right\| \leqslant 1 / M$ for every $x$. Hence

$$
\int_{0}^{\infty} \inf _{\|x\|<t} \frac{d t}{\left\|\left[F^{\prime}(x)\right]^{-1}\right\|} \geqslant \int_{0}^{\infty} M d t=\infty
$$

So by Theorem 3.2, $F$ is a diffeomorphism of $X$ onto $Y$.

The next corollary pertains to a class of maps that are related to quasi-conformal maps.

CoRollary 3.2. Suppose $F: X \rightarrow Y \in C^{1}(X)$ and $F^{\prime}(x)$ is invertible $\forall x \in$ $X$. Also suppose that there are continuous, positive nondecreasing real-valued functions $M(t), \bar{M}(t)$ so that

$$
\left\|F^{\prime}(x)\right\| \leqslant M(\|x\|) \text { and }\left\|\left[F^{\prime}(x)\right]^{-1}\right\| \leqslant \bar{M}(\|x\|) \text {. }
$$

Then if $M(t) \bar{M}(t) \leqslant \lambda(t)$ for all real $t$ where $\lambda(t)>0$ and $\int_{0}^{\infty} 1 / \lambda(t) d t=\infty$, $F$ is a diffeomorphism of $X$ onto $Y$. 
Proof.

$$
\begin{aligned}
\left\|F^{\prime}(0)\right\|\left\|\left[F^{\prime}(y)\right]^{-1}\right\| & \leqslant \sup _{\|z\| \leqslant\|y\|}\left\|F^{\prime}(z)\right\| \cdot \sup _{\|z\| \leqslant\|y\|}\left\|\left[F^{\prime}(z)\right]^{-1}\right\| \\
& \leqslant M(\|y\|) \bar{M}(\|y\|) \leqslant \lambda(\|y\|) .
\end{aligned}
$$

Thus

$$
1 /\left\|\left[F^{\prime}(y)\right]^{-1}\right\| \geqslant\left\|F^{\prime}(0)\right\| / \lambda(\|y\|) .
$$

So by virtue of Theorem 3.2, $F$ is a diffeomorphism of $X$ onto $Y$.

Corollary 3.3. Let $H$ be a Hilbert space and $F: H \rightarrow H \in C^{1}(H)$. Furthermore suppose there is a positive real-valued function $\lambda(x)$ such that $\left(F^{\prime}(x) z, z\right)$ $\geqslant \lambda(x)\|z\|^{2}$. Then if $\int_{0}^{\infty} \inf _{\|x\|<t} \lambda(x) d t=\infty, F$ is a diffeomorphism of $H$ onto itself.

Proof. By the Lax-Milgram theorem, $F^{\prime}(x)$ is invertible and $\left\|\left[F^{\prime}(x)\right]^{-1}\right\| \leqslant$ $1 / \lambda(x)$. Thus $F$ is a local diffeomorphism. Also

$$
\int_{0}^{\infty} \inf _{\|x\| \leqslant t} \frac{1}{\left\|\left[F^{\prime}(x)\right]^{-1}\right\|} d t \geqslant \int_{0}^{\infty} \inf _{\|x\| \leqslant t} \lambda(x) d t=\infty .
$$

Hence Theorem 3.2 is applicable.

4. Global homeomorphisms between finite-dimensional spaces. For $X=\mathrm{R}^{N}$, we have the following slightly stronger version of Theorem 3.1.

THEOREM 4.1. Let $h(s)=\inf _{\|x\|=s} B(x)$. If $\int_{0}^{\infty} h(s) d s=\infty$, then $\mathbf{R}^{N}$ is complete with respect to arc length with weight $B$.

Proof. Let $P(t) \in C^{1}[0, b)$ and suppose $L_{0}^{b}(P)<\infty$. Let $0<\delta<b$. Following the proof given in Theorem 3.1, we have that $\infty>\int_{\|P(0)\|}^{\|P(\delta)\|} h(s) d s$. Hence $\{P(t)\}_{0<t<b}$ is bounded. Since $B(x)$ is a continuous, real-valued function on $\mathbf{R}^{N}$, it maps bounded sets into bounded sets. Thus $B(P(t))$ is bounded from below by some positive number (since $B(x)$ is positive). Again, as in Theorem 3.1, we find that if $t_{i} \rightarrow b$, then

$$
\sum_{i=1}^{n}\left\|P\left(t_{i+1}\right)-P\left(t_{i}\right)\right\| \leqslant \frac{1}{\lambda} \int_{0}^{b} B(P(t))\left\|P^{\prime}(t)\right\| d t<\infty,
$$

and thus we conclude that $\lim _{t \rightarrow b} P(t)$ exists and is finite.

THEOREM 4.2. Suppose $F: \mathbf{R}^{N} \rightarrow \mathbf{R}^{N}, F \in C^{1}\left(\mathbf{R}^{N}\right)$ and also $F^{\prime}(x)$ is invertible for all $x \in \mathbf{R}^{N}$. If

$$
\int_{0}^{\infty} \inf _{\|x\|=t}\left(1 /\left\|\left[F^{\prime}(x)\right]^{-1}\right\|\right) d t=\infty,
$$

then $F$ is a diffeomorphism of $\mathbf{R}^{N}$ onto itself. 
Proof. The proof mimics that of Theorem 3.2, except that we use Theorem 4.1 in place of Theorem 3.1.

Lemma 4.1. Let $L: \mathbf{R}^{N} \rightarrow \mathbf{R}^{N}$ be an invertible linear operator. Then $|\operatorname{det} L|\left|\left(L^{-1} x, y\right)\right| \leqslant\|x\|\|y\|\|L\|^{n-1}(n-1)^{-(n-1) / 2}$ for all $x, y \in \mathbf{R}^{N}$.

Proof. See [7, Part II, p. 1020].

CoRollary 4.1. Let $F: \mathbf{R}^{N} \rightarrow \mathbf{R}^{N} \in C^{1}\left(\mathbf{R}^{N}\right)$. Suppose that (i) $\left|\operatorname{det} F^{\prime}(x)\right|$ $>\alpha>0$, and (ii) $\left\|F^{\prime}(x)\right\| \leqslant M$. Then $F$ is a diffeomorphism of $\mathbf{R}^{N}$ onto $\mathbf{R}^{N}$.

Proof. From Lemma 4.1, we have that

$$
\left|\operatorname{det} F^{\prime}(x)\right|\left|\left(\left[F^{\prime}(x)\right]^{-1} z, w\right)\right| \leqslant c(n)\|z\|\|y\|\left\|F^{\prime}(x)\right\|^{n-1},
$$

where $c(n)=(n-1)^{-(n-1) / 2}$. Choose $z$ so that $\|z\|=1$, and let $w=$ $\left[F^{\prime}(x)\right]^{-1} z$. With these choices, (1) becomes

$$
\left|\operatorname{det} F^{\prime}(x)\right|\left\|\left[F^{\prime}(x)\right]^{-1} z\right\|^{2} \leqslant c(n)\left\|\left[F^{\prime}(x)\right]^{-1} z\right\|\left\|F^{\prime}(x)\right\|^{n-1} .
$$

Using hypotheses (i) and (ii) we have that

$$
\left\|\left[F^{\prime}(x)\right]^{-1} z\right\| \leqslant c(n) M^{n-1} / \alpha, \text { for all }\|z\|=1 .
$$

Hence

$$
\left\|\left[F^{\prime}(x)\right]^{-1}\right\| \leqslant c(n) M^{n-1} / \alpha, \text { for each } x .
$$

Thus, by Corollary 3.1, $F$ is a diffeomorphism of $\mathbf{R}^{N}$ onto $\mathbf{R}^{N}$.

Corollary 4.2. Suppose $F: \mathbf{R}^{N} \rightarrow \mathbf{R}^{N}$ is continuously differentiable. Also, suppose that (i) $\left|\operatorname{det} F^{\prime}(x)\right|>\alpha>0$, and (ii) is quasi-conformal, i. e., $\mathrm{G} M \ni$ $\left\|F^{\prime}(x)\right\|\left\|\left[F^{\prime}(x)\right]^{-1}\right\| \leqslant M$, for all $x$. Then $F$ is a diffeomorphism of $\mathbf{R}^{N}$ onto $\mathbf{R}^{N}$.

Proof. By Lemma 4.1, we have, with $c(n)=(n-1)^{-(n-1) / 2}$,

$$
\left|\operatorname{det} F^{\prime}(x)\right|\left|\left(\left[F^{\prime}(x)\right]^{-1} z, w\right)\right| \leqslant c(n)\|z\|\|w\|\left\|F^{\prime}(x)\right\|^{n-1} .
$$

Choosing $z=w$ and $\|z\|=1$, (1) becomes

(2) $\left|\operatorname{det} F^{\prime}(x)\right|\left|\left(\left[F^{\prime}(x)\right]^{-1} z, z\right)\right| \leqslant c(n)\left\|F^{\prime}(x)\right\|^{n-1}, \quad \forall\|z\|=1$.

Taking $\sup _{\|z\|=1}$ of the left side of (2), we get $\left|\operatorname{det} F^{\prime}(x)\right|\left\|\left[F^{\prime}(x)\right]^{-1}\right\| \leqslant c(n)\left\|F^{\prime}(x)\right\|^{n-1}$.

Using hypotheses (i) and (ii), we have

$$
\left\|\left[F^{\prime}(x)\right]^{-1}\right\| \leqslant\left[c(n) M^{n-1} / \alpha\right]^{1 / n} \text { for all } x \text {. }
$$


By Corollary 3.1, $F$ is a diffeomorphism of $\mathbf{R}^{N}$ onto $\mathbf{R}^{N}$.

Corollary 4.2 is true under the weaker hypothesis $\operatorname{det} F^{\prime}(x) \neq 0$, provided $N \geqslant 3[16]$.

In general, the hypotheses of Theorems 3.2 and 4.2 cannot be entirely omitted as the following examples show:

EXAMPLE 4.1. $F(x)=F\left(x_{1}, \cdots, x_{n}\right)=\left(\tan ^{-1} x_{1}, x_{2}\left(1+x_{1}^{2}\right)^{2}, x_{3}, \cdots, x_{n}\right)$ is a $C^{1}$ map of $R^{n}$ into $R^{n}$ and

$$
F^{\prime}(x)=F^{\prime}\left(x_{1}, \cdots, x_{n}\right)=\left|\begin{array}{cc|c}
\frac{1}{1+x_{1}^{2}} & 4 x_{1} x_{2}\left(1+x_{1}^{2}\right) & 0 \\
0 & \left(1+x_{1}^{2}\right)^{2} & \\
\hline 0 & I_{n-2}
\end{array}\right| .
$$

Since $\operatorname{det} F^{\prime}(x)=x_{1}^{2}+1>1, F$ satisfies the conditions of the inverse function theorem. By looking at the characteristic polynomial of $F^{\prime}(x)$, we see that $\lambda=1 /\left(1+x_{1}^{2}\right)$ is an eigenvalue, and so $1 / \lambda=1+x_{1}^{2}$ is an eigenvalue of $\left[F^{\prime}(x)\right]^{-1}$. Therefore $\left\|\left[F^{\prime}(x)\right]^{-1}\right\| \geqslant 1+x_{1}^{2}$, and so we have that

$$
\inf _{\|x\|<t} \frac{1}{\left\|\left[F^{\prime}(x)\right]^{-1}\right\|} \leqslant 1 /\left(1+t^{2}\right) \text {. }
$$

Since $\int_{0}^{\infty} d t /\left(1+t^{2}\right)<\infty, F$ does not satisfy the hypotheses of Theorems 3.2 and 4.2 and we observe that $F$ is one-one, but not onto.

EXAMPLE 4.2. A second example of a univalent map which is not onto is the famous example of Fatou and Bieberbach [3, p. 45]. This is an example of an analytic map $F$ of $\mathbf{C}^{2}$ into itself whose Jacobian (of the map considered as mapping $\mathbf{R}^{4} \rightarrow \mathbf{R}^{4}$ ) is identically equal to 1 and $F$ is univalent; however, the range of $F$ omits a full open neighborhood of a point in $\mathbf{C}^{2}$.

EXAMPLE 4.3. $F\left(x_{1}, x_{2}\right)=e^{x_{1}}\left(\cos x_{2}, \sin x_{2}\right) . F$ is a $C^{1}$ map of $\mathbf{R}^{2} \rightarrow$ $\mathbf{R}^{2}$ which is neither one-one nor onto (it omits 0 ). We observe that $\left\|F^{\prime}\left(x_{1}, x_{2}\right)\right\|=$ $e^{x_{1}}$, thus $1 /\left\|\left[F^{\prime}\left(x_{1}, x_{2}\right)\right]^{-1}\right\| \leqslant e^{x_{1}}$. So

$$
\inf _{\|x\|<t} \frac{1}{\left\|\left[F^{\prime}\left(x_{1}, x_{2}\right)\right]^{-1}\right\|} \leqslant e^{-t} \text { and } \int_{0}^{\infty} e^{-t} d t<\infty .
$$

Examples 4.1 and 4.2 also show that the condition $\left|\operatorname{det} F^{\prime}(x)\right| \geqslant \alpha>0$ is not in itself sufficient to insure that $F$ is a global homeomorphism, for if $F\left(x_{1}, x_{2}\right)=\left(\tan ^{-1} x_{1}, x_{2}\left(1+x_{1}^{2}\right)^{2}, x_{3}, \cdots, x_{n}\right)$ then $\operatorname{det} F^{\prime}\left(x_{1}, x_{2}\right)>1$, yet $F$ is not a homeomorphism of $\mathbf{R}^{n}$ onto $\mathbf{R}^{n}$. However it is known [8] that if $F$ is a gradient map of $\mathbf{R}^{2}$ into itself, then $\operatorname{det} F^{\prime}(x)<\alpha<0$ insures that $F$ is globally one-one.

5. Conclusion. If we inspect the methods that were employed in determining when a mapping between two Banach spaces is a homeomorphism, we see that 
the properties of global univalence and surjectivity were related in an intimate way, i. e., once we showed that the map $F: X \rightarrow Y$ is a covering space map of the simply connected space $Y$, then $F$ was automatically univalent by Lemma 1.1. In the general case of a mapping $F$ of some domain $D$ into a Banach space we usually have little information about $F(D)$. Thus the assumption that $F(D)$ is simply connected is not viable and so Lemma 1.1 is not applicable. In subsequent papers we shall investigate the questions of global univalence and surjectivity independently and without any assumptions on the range of the mapping at hand.

\section{REFERENCES}

1. S. Banach and S. Mazur, Über mehrdeutige stetige abbildungen, Studia Math. 5 (1934), 174-178.

2. M. S. Berger and M. S. Berger, Perspectives in nonlinearity. An introduction to nonlinear analysis, Benjamin, New York, 1968. MR 40 \#4971.

3. S. Bochner and W. T. Martin, Several complex variables, Princeton Math. Series, vol. 10, Princeton Univ. Press, Princeton, N. J., 1948. MR 10, 366.

4. F. E. Browder, Covering spaces, fiber spaces and local homeomorphisms, Duke Math. J. 21 (1954), 329-336. MR 15, 978.

5. R. Cacciopoli, Sugli eleminti uniti delle transformazioni funzionali, Rend. Sem. Mat. Univ. Padova 3 (1932), 1-15.

6. H. Cartan, Sur les transformations localement topologiques, Acta Litt. Sci. Szeged 6 (1933), 85-104.

7. a) N. Dunford and J. T. Schwartz, Linear operators. I: General theory, Pure and Appl. Math., vol. 7, Interscience, New York, 1958. MR 22 \#8302.

b) Linear operators. II: Spectral theory. Self-adjoint operators in Hilbert space, Interscience, New York, 1963. MR 32 \#6181.

8. N. V. Efimov, Differential criteria for homeomorphisms of certain mappings with applications to the theory of surfaces, Mat. Sb. 76 (118) (1968), 499-512 = Math. USSR Sb. 5 (1968), 475-488. MR 37 \#5821.

9. W. B. Gordon, On the diffeomorphisms of Euclidean space, Amer. Math. Monthly 79 (1972), 755-759.

10. J. Hadamard, Sur les transformations ponctuelles, Bull. Soc. Math. France 34 (1904), $71-84$.

11. P. Hartman, Ordinary differential equations, Wiley, New York, 1964. MR 30 \#1270.

12. F. John, On quasi-isometric mappings. I, Comm. Pure Appl. Math. 21 (1968), 77110. MR 36 \#5716.

13. M. A. Lavrent'ev, Sur une critère differentiel des transformations homéomorphes des domaines à trois dimensions, Dokl. Akad. Nauk SSSR 22 (1938), 241-242.

14. P. Levy, Sur les fonctions des lignes implicites, Bull. Soc. Math. France 48 (1920), 13-27.

15. R. Plastock, A note on surjectivity of nonlinear maps (manuscript). 
16. V. A. Zorič, $A$ theorem of M. A. Lavrent'ev on quasiconformal space maps, Mat. Sb. 74 (116) (1967), 417-433 = Math. USSR Sb. 3 (1967), 389-403. MR 36 \#6617.

17. R. Hermann, Differential geometry and the calculus of variations, Math. in Sci. and Engineering, vol. 49, Academic Press, New York, 1968. MR 38 \#1635.

DEPARTMENT OF MATHEMATICS, STATEN ISLAND COMMUNITY COLLEGE, NEW YORK, NEW YORK 10301

Current address: Division of Science and Mathematics, The Cooper Union, Cooper Square, New York, New York 10003 